\title{
A DATA MINING STUDY OF SINDEC COMPLAINTS IN THE PERIOD 2013-2017
}

\author{
Vagner Sargiani ${ }^{1}$, Leandro Nunes de Castro $^{2}$ and Leandro Augusto da Silva ${ }^{1}$ \\ ${ }^{1}$ Mackenzie Presbyterian University, Brazil \\ ${ }^{2}$ Natural Computing and Machine Learning Laboratory \\ Graduate Program in Electrical Engineering and Computing \\ Mackenzie Presbyterian University, Brazil
}

\begin{abstract}
The SINDEC's complaints database is an important source of information about the sectors and problems that cause complaints in Brazil. By analyzing these data, one can understand the main complaints and whether companies and institutions are working to solve such problems. The objective of this work is to apply data mining techniques, more specifically descriptive analyses and a classification algorithm, to extract knowledge from the data over the period 2013 and 2017.
\end{abstract}

\section{KEYWORDS}

SINDEC data, Data Mining, Descriptive analysis, Decision Tree, J48

\section{INTRODUCTION}

The relationship between consumers and businesses, from the beginning, has always been unilateral: companies developed products or services that they believe consumers want or need. However, companies and institutions were not used to have feedback from consumers over what was being offered, and if a consumer suffered any damage or had problems with the product or service. There was no mechanism to present and investigate complaints. This has changed with the creation of services aimed at receiving consumer complaints, such as PROCON, Reclame Aqui and others. According to [Bessa and de Moura 2014], these services began to be created by law in the 1970s, with the emergence of the first consumer protection agencies. In the 1980s this was intensified with the creation of the National Consumer Advocacy Council and PROCONs. With this, consumers have gained public (government) channels to register their complaints and, more recently, private initiatives, such as Reclame Aqui, have emerged.

The information about complaints stored over time, has allowed companies to improve their products and services. But this information also allows us to draw a picture of which services, or companies, have had the most complaints and if they are improving or worsening over time. With the use of data mining techniques, several insights can be obtained from such information. This paper performs a descriptive analysis and employs a decision tree to provide insights out of the main complaint data provided by SINDEC ${ }^{1}$.

The paper is organized as follows. Section 2 brings a briefing on data mining just to pave the ground for the analysis to be presented in Section 3. The work is concluded in Section 4 with a discussion and some proposals for future investigations.

\footnotetext{
${ }^{1}$ National Consumer Defense Information System (Sistema Nacional de Informações de Defesa do Consumidor in Portuguese).
} 


\section{A BRIEFING ON DATA MINING}

The terminology data mining was created as a metaphor to the process of mining precious metals, where mining represents the extraction of information from databases, and the precious metal is the knowledge obtained, which is valuable for decision making [de Castro and Ferrari 2015; Silva et al. 2016]. It corresponds to the use of automatic or semi-automatic processes to analyze data. The process can be described succinctly according to Figure 1, where data is extracted from the database, pre-processes, mined and the results validated.

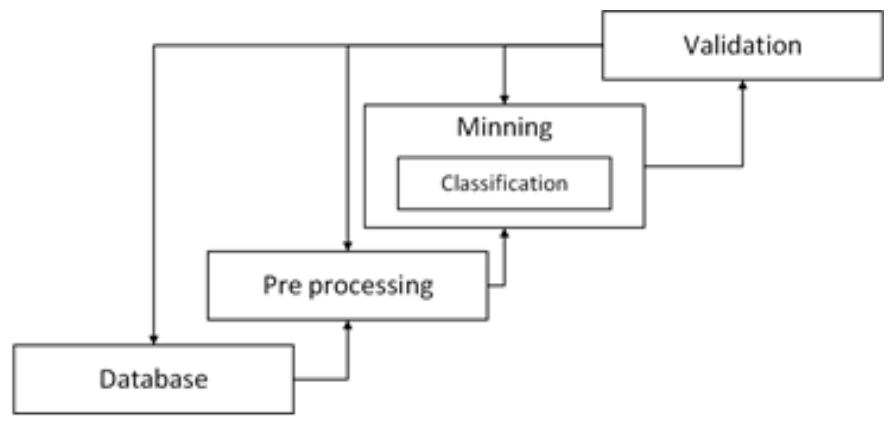

Figure 1. The data mining process. Source: de Castro and Ferrari 2015, pg. 6

The possible data mining operations will depend on the data to be processed, and if they have an attribute that defines the result obtained for the data (called class or target). For example, in text clustering there is usually no target attribute, and the initial goal is to cluster texts so that similar documents belong to the clusters [Jo et al. 2017].

The data pre-processing phase makes the necessary data preparation so that knowledge can be extracted from it. This preparation will be based on the format in which the data is saved, and what are the desired results. In [Dwivedi and Rawat 2016], the authors describe different pre-processing techniques when the purpose is to analyze various web information (web mining), and the sources can be diverse, such as text files, numeric data, images, video, audio, among others.

For text data, some of the standard pre-processing include the removal of accents and non-graphic characters. It may also be necessary to remove punctuation and adjust letter case [Jo et al. 2017]. Text data may also represent categories, and in some cases, it may be necessary to structure text data as numeric data.

Numeric data can usually be of two types: continuous, when an infinite number of values can be observed for each attribute; and discrete, when a finite, countable, number of values can be observed for each attribute.

The data analysis task can be divided into two different types: descriptive analysis, which characterize the general properties of the data; and predictive analysis, which make inferences from the data aiming at predictions. These types of analyses will be presented in the following, detaching the investigation that will be made in the present paper.

\subsection{Descriptive Analysis}

After pre-processing, it is necessary to understand how this information is distributed and organized. In the descriptive analysis, the data types and their distributions are obtained [de Castro and Ferrari 2015, Bevington and Wolcott 1996]. The most commonly used descriptive analyses include the average, median, midpoint, mode, standard deviation, variance, and frequency distribution. These measures are important for a first understanding of the data in the database.

\subsection{Predictive Analysis}

When the objects have a target attribute it is possible to use them for designing a predictor, that is, to train a machine learning algorithm so that it can predict the target values of unseen data. When the target attribute is discrete, one can design a classifier, which is a model generated based on the data presented during the 
training phase and that can be used to predict to which class a new object belongs to according to its attributes [de Castro and Ferrari 2015]. It can also be described as the discovery of the relationship between objects of a set of data, described by a series of characteristics and the labels associated with them [Silva et al. 2016].

There are several classification approaches that address the problem in different ways, such as decision trees [Samsani 2016], Bayesian algorithms [Claster et al. 2010], nearest neighbors [Qureshi et al. 2015], and neural networks [Nazzal et al. 2008].

The present paper focuses only on the use of decision trees as a classifier for the complaint data. Decision trees use the training data to build a hierarchical structure similar to a tree, where each node represents an attribute, and the branches represent one of the possible values for this attribute. The leaves of the tree correspond to the target values. According to [Patil and Sherekar 2013], the decision tree algorithm named $\mathrm{J} 48$ uses the simple C4.5, which is an extension of the ID3 algorithm [Ahishakiye et al. 2017], [Quinlan 1986], to create a binary tree, that is, a tree where each node has, at most, two children. The use of the J48 algorithm is interesting because if there are null attributes, they are ignored during the training process. Also, the J48 algorithm allows pruning the generated tree, thus reducing its complexity.

\subsection{Performance Evaluation}

The performance evaluation of data mining algorithms aims at assessing the effectiveness of the models generated. There are several methods for evaluating the performance of algorithms and some of them will be discussed here focusing on classification tasks [Rodriguez et al. 2010, Alippi and Roveri 2010, Nurhayati et al. 2014]. In such cases, the datasets are usually divided into training and test set, so that the former is used to train the classifier, and the latter is used to estimate its performance when in production [Samsani 2016, Ciaburro and Venkateswaran 2017, Silva et al. 2008]. The first phase is called training, and the last one is called testing or validation.

One way to fully present the performance of a binary classification algorithm (that is, the one with only two possible classes, such as positive and negative) is to build a matrix that relates the desired (target) classes with the predicted ones. This confusion matrix, also known as contingency matrix or error matrix, has in the rows the objects in the original classes and in the columns the objects in the predicted classes. Based on the values presented in the binary problem confusion matrix, there are two important rates, usually expressed as percentage values: the true positive rate (TPR) and the false positive rate (FPR):

$$
\begin{gathered}
T P R=\frac{T P}{T P+F N} \\
F P R=\frac{F P}{F P+T N}
\end{gathered}
$$

where $T P$ is the number of true positives, $F N$ is the number of false negatives, $F P$ is the number of false positives, and $T N$ is the number of true negatives.

The overall success rate of the algorithm, known as accuracy (ACC), is the number of correct ratings divided by the total number of ratings, and the error $(E)$ is the inverse of the accuracy:

$$
\begin{aligned}
A C C= & \frac{T P+T N}{T P+F P+T N+F N} \\
& E=1-A c c
\end{aligned}
$$

Two other measures of great practical relevance for performance evaluation are precision ( $\mathrm{Pr})$ and recall $(\mathrm{Re})$. These two measures are associated with the concept of relevance, where precision measures the quality or accuracy of the algorithm, while recall measures its completeness:

$$
\begin{aligned}
& P r=\frac{T P}{F P+T P} \\
& R e=\frac{T P}{F N+T P}
\end{aligned}
$$


Using these two measures, we can calculate a very common performance measure in data mining, called $\mathrm{F}$-measure (or $\mathrm{F}$-score), which is a harmonic mean and considers precision and recall, and is contained in the interval $[0,1]$, with 0 being the worst result and 1 indicating a perfect compromise between Precision and Recall:

$$
F=\frac{2 \times P r \times R e}{(P r+R e)}
$$

To evaluate classifiers, one can also use the Kappa statistic, which measures the agreement of two evaluators, with each classifying $n$ objects and $C$ mutually exclusive classes:

$$
\begin{aligned}
\kappa & =\frac{\operatorname{Pr}(a)-\operatorname{Pr}(e)}{1-\operatorname{Pr}(e)} \\
\operatorname{Pr}(a) & =\frac{T P+T N}{N} \\
\operatorname{Pr}(e) & =\left(\frac{T P+F N}{n} \times \frac{T P+F P}{n}\right)+\left(\frac{F P+T N}{n} \times \frac{F N+T N}{n}\right)
\end{aligned}
$$

where $\operatorname{Pr}(a)$ is the relative agreement observed between the evaluators, and $\operatorname{Pr}(e)$ is the hypothetical probability of a random agreement and $n$ (total of objects). When the evaluators fully agree, $\kappa=1$, if there is no agreement, $\kappa=0$.

\section{MINING THE SINDEC DATA}

This section starts by describing the data to be analyzed and follows with the methodology used in the analyses. Then, the data and main results are described and discussed.

\subsection{Dataset and Main Research Questions}

The database used in this paper was initially found in the website Kaggle [Gerosa 2017], with a brief description of the content and a link to the original source [Ministério da Justiça 2019]. When comparing the data available in Kaggle with that of the original source, the latter was chosen because it is more updated (last update in 01/25/2019). The period that will be used in the experiments is from 2013 to 2017, as this includes the most recent data available. As this database has complaints from the most diverse business sectors, it is not possible to evaluate the whole dataset. For this reason, one of the steps will be to rank the sectors, allowing the generation of a sample that will be used to train the decision tree algorithm.

The complaints dataset has a large variety of economic sectors (identified by the attributes with acronym $\mathrm{CNAE}^{2}$ ) whose modes of operation are distinct, and consequently the reported problems are also different. The first analysis to be performed is to generate the frequency distribution of the main attributes. This will allow us to understand important details of each variable and the behavior of complaints, sampling the data based on the frequency distributions. The main research question to be answered is as follows: Is it possible to predict whether, if the sector and subject are chosen, a complaint about a problem will be addressed? To answer this question, a decision tree will be trained and the rules created analyzed.

\subsection{Methodology}

The complaints data includes texts to be processed and analyzed. After some basic text pre-processing, such as the removal of accents, quotation marks and replacing all letters by small caps, a sampling method based on the CNAE will be used to select the data to train the decision tree. To perform the classification, we used a

\footnotetext{
${ }^{2}$ Classificação Nacional de Atividades Econômicas, in English: National Classification of Economic Activities
} 
decision tree implemented in the J48 algorithm. To evaluate performance, a $k$-fold cross validation process with $k=10$, was used. After training, the confusion matrix will be generated with the results obtained for the test data.

The experiments will be performed using the following tools for analysis:

- Notepad ++: This was the text editor chosen for manipulating the raw files before the import process by R;

- R Studio: We will use R Studio to treat the database and generate the descriptive data, in addition to the subsets of data for analysis;

- Weka: The Weka will be used to perform the experiments.

\subsection{Data Description}

The beginning of the process is the download of the data files containing the consumer complaints for the period from 2013 to 2017. The files were adjusted before being imported into the R Studio analytic tool. With the imported files, character cleanup, font size change, and necessary conversions were made. The resulting database has 23 attributes, described in Table 1. Some attributes identified in column Used as "No" will not be used in the present experiments and will be removed from the dataset. The 11 attributes chosen are detached in light gray.

Table 1. Data dictionary

\begin{tabular}{|c|c|c|c|}
\hline Field & Data Type & Description/Values & Used? \\
\hline AnoCalendario & Integer & Extraction Year & No \\
\hline DataArquivamento & Date/Time & Date the record was archived & No \\
\hline DataAbertura & Date/Time & Date the record was created & No \\
\hline CodigoRegiao & Nominal Integer & Region ID & No \\
\hline Regiao & Nominal Text & Region Description & Yes \\
\hline UF & Textual & State Abbreviation & Yes \\
\hline strRazaoSocial & Textual & Corporate Name (free text) & No \\
\hline strNomeFantasia & Textual & Trade Name (free text) & No \\
\hline Tipo & Nominal Integer & 1 - Legal Entity (CNPJ); 0 - Individuals (CPF) & No \\
\hline NumeroCNPJ & Numeric & CPF or CNPJ & No \\
\hline RadicalCNPJ & Numeric & For CNPJ, it identifies branch of service & No \\
\hline RazaoSocialRFB & Textual & Corporate Name when CNPJ is registered & No \\
\hline NomeFantasiaRFB & Textual & Trade Name when CNPJ is registered & No \\
\hline CNAEPrincipal & Nominal Numeric & ID for the field (DescCNAEPrincipal) - 961 Ids & Yes \\
\hline DescCNAEPrincipal & Nominal Text & 944 Descriptions for different CNAE & Yes \\
\hline Atendida & Nominal Text & Attendance: $\mathrm{S}$ - Answered; N-NOT Answered & Yes \\
\hline CodigoAssunto & Nominal Integer & Ids for the field (DescricaoAssunto) - 240 Ids & Yes \\
\hline DescricaoAssunto & Nominal Text & 222 descriptions for diferent subjects & Yes \\
\hline CodigoProblema & Nominal Integer & Ids for the field (DescricaoProblema) - 2385 Ids & Yes \\
\hline DescricaoProblema & Nominal Text & 208 descriptions for different problems & Yes \\
\hline SexoConsumidor & Nominal Text & M - Male; F-Female; N-Not applicable & Yes \\
\hline FaixaEtariaConsumidor & Nominal Text & 7 ranges that represent ages +2 of support & Yes \\
\hline CEPConsumidor & Numeric & Zip code of the consumer who filed the complaint & No \\
\hline
\end{tabular}

For the selected attributes, a number of graphs were generated to better understand the database information. For the attributes CNAE, Subject and Problem, we generated the frequency distribution graphs for the 15 highest frequencies (Figures 2, 3 and 4). For the other attributes, sector graphs were generated, since the number of items is smaller. 


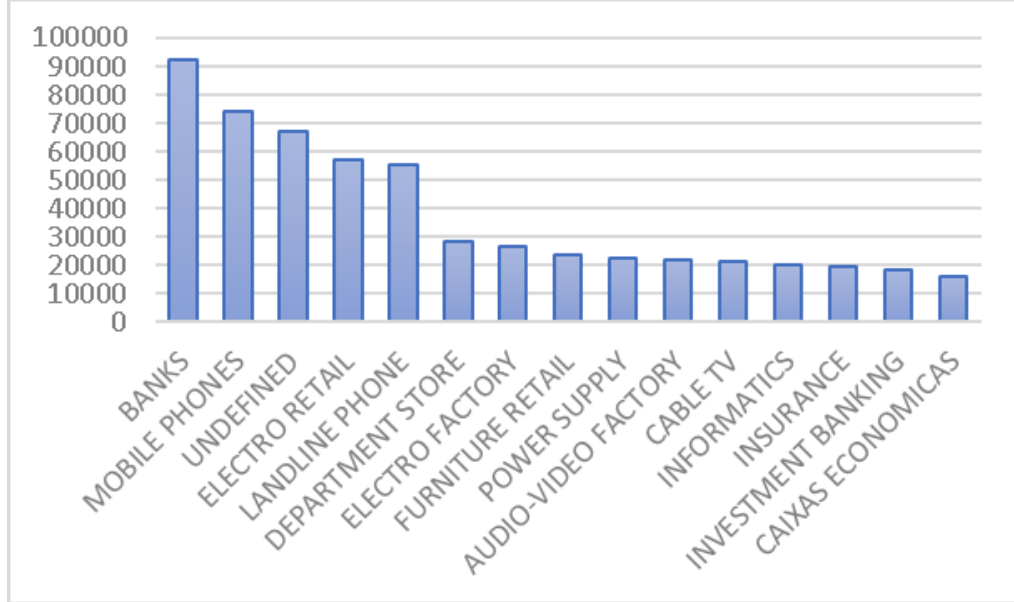

Figure 2. Frequency distribution of the market sectors

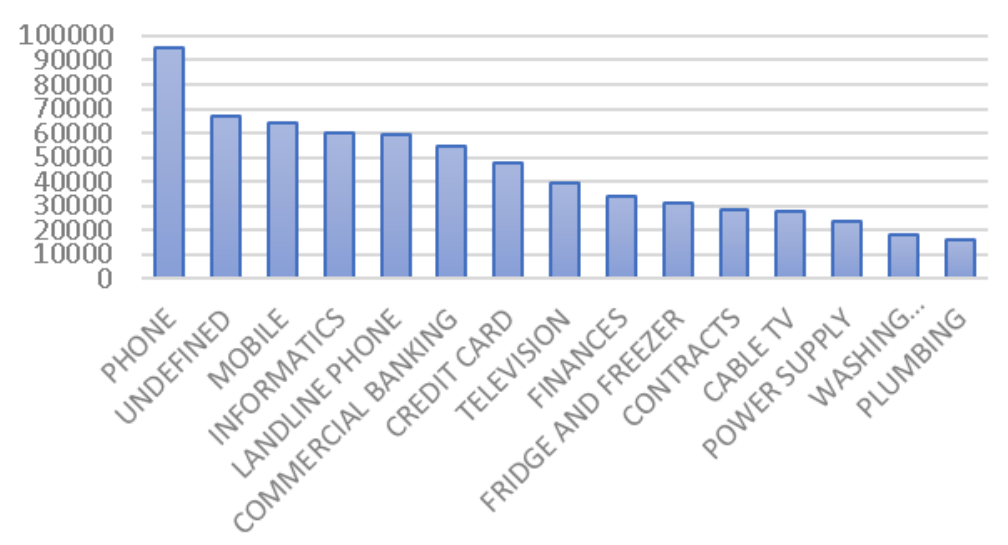

Figure 3. Frequency distribution of the most covered subjects

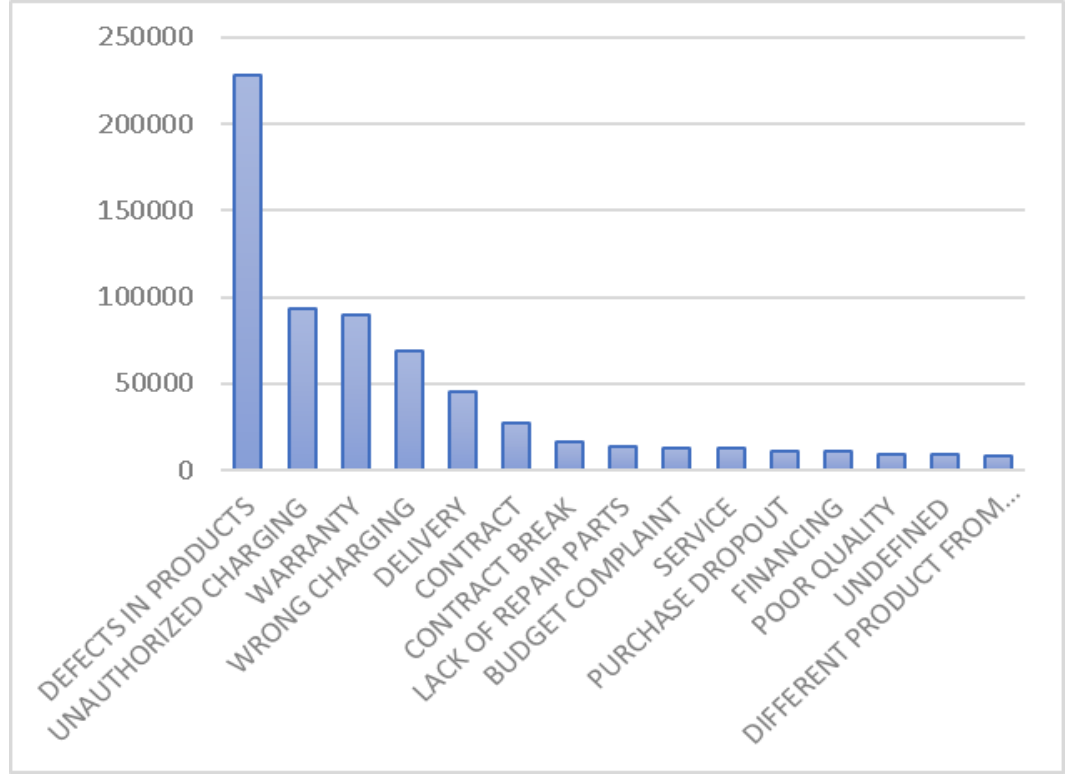

Figure 4. Frequency distribution of the most frequently addressed problems 

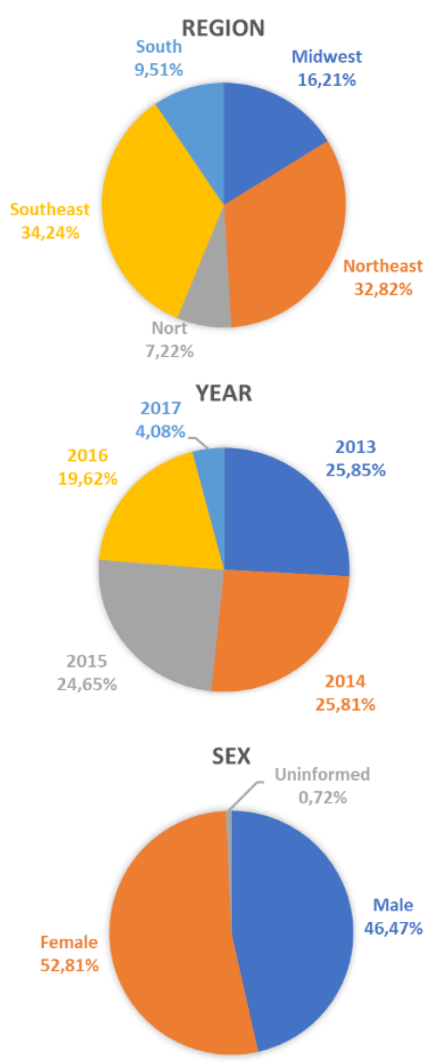
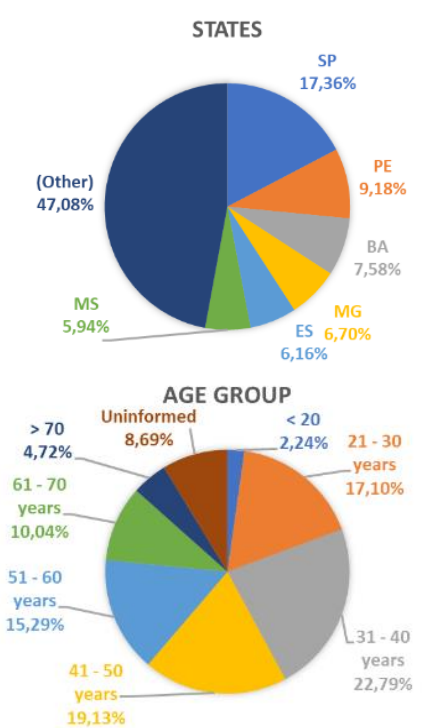

ANSWERED

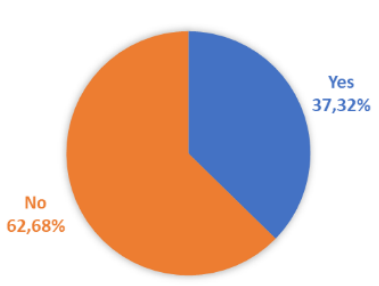

Figure 5. Frequency distributions by Region, State, Year, Age Group, Sex and Answered

Based on the data shown in the frequency distribution graph of Figure 2, the mode of the CNAE is related with BANKS. This value was used to filter the database, resulting in a smaller dataset with 92,367 records, where it was possible to generate the frequency distribution for the subjects of this CNAE. The idea is to put a lens in the most complained market sector. A new frequency distribution graph was generated and illustrated in Figure 6. By looking at this data we observe that retail banks have the most number of complaints, followed by credit cards, financial institutions and the contracts.

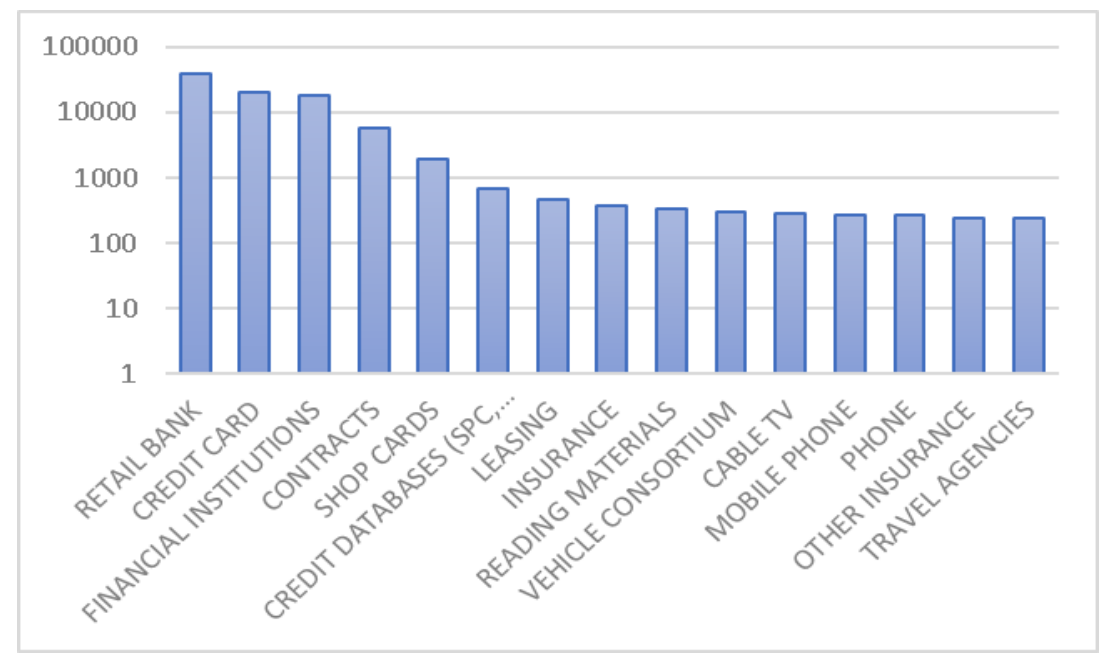

Figure 6. Frequency distribution of the most approached subjects for the BANKS market sector 
Again, we placed another lens on RETAIL BANK to uncover the most frequently addressed problems (The figure 7), and observed that unauthorized charging is by far the worst complaint.

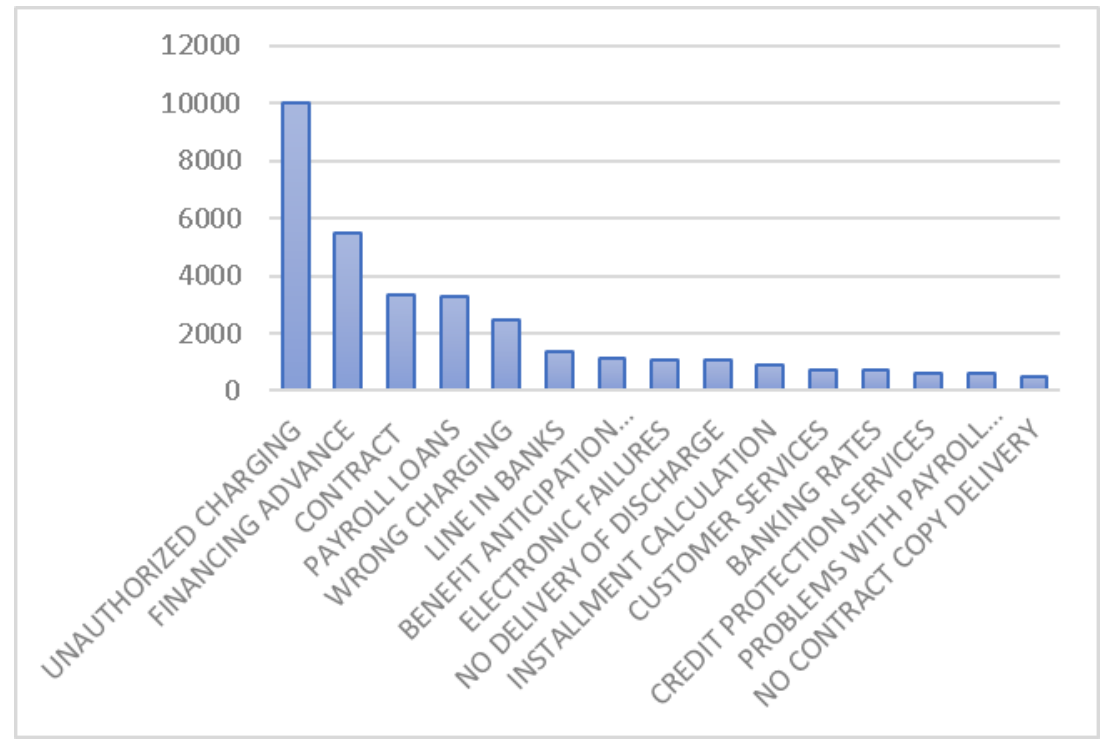

Figure 7. Frequency distribution graph of the 15 most frequently addressed problems for the Retail Bank

Finally, the new generated data subset with $\mathbf{3 8 , 3 1 3}$ objects was used to train the decision tree, as will be described in the following.

\subsection{Decision Tree}

The dataset containing the previously filtered records was imported into the Weka tool. The Classify tab was selected and the J48 algorithm was chosen as the classifier. The parameters used were: confidenceFactor $=0.25$, minNumObj $=2$ and unpruned $=F A L S E$; for all other parameters the default values were maintained. The choice to work with a cut tree (with prune) was made because, in the tests performed, the cut tree had a better overall result, besides presenting a smaller volume of nodes and leaves. The validation chosen was cross-validation with $k=10$ folds. The target attribute chosen was "Atendida".

With the algorithm parametrized, the decision tree training was performed. The time for constructing the model was 0.67 seconds and, after training, the confusion matrix (Table 2) was generated and the results presented in Table 3 were obtained.

Table 2. Confusion Matrix

\begin{tabular}{|l|l|l|}
\hline & $N$ & $S$ \\
\hline$N$ & 11,236 & 6,714 \\
\hline$S$ & 5,993 & 14,370 \\
\hline
\end{tabular}


Table 3. Training: Decision tree

\begin{tabular}{llc}
\hline Decision Tree Results & & \\
\hline Indicator & Value & Percentage \\
\hline Total Number of Instances & 38,313 & - \\
\hline Number of Leaves & 1,151 & - \\
\hline Size of the tree & 1,421 & - \\
\hline Correctly Classified Instances (Eq.3) & 25,606 & $66.83 \%$ \\
\hline Incorrectly Classified Instances & 12,707 & $33.17 \%$ \\
\hline Kappa statistic (eq.8) & 0.3324 & - \\
\hline Mean absolute error & 0.4041 & - \\
\hline Root mean squared error & 0.4606 & - \\
\hline Relative absolute error & 0.8113 & - \\
\hline Root relative squared error & 0.9230 & - \\
\hline
\end{tabular}

The Kappa statistic was 0.3324 , indicating that the agreement between the values is low but has some significance (it is not pure chance).

Using the data from the confusion matrix it is possible to calculate the TPR (Eq. 1), FPR (Eq. 2), Precision (Eq. 5) and Recall (Eq. 6): TPR $=62,60 \%$, FPR $=29,43 \%, \operatorname{Pr}=65,22 \%$, and $\operatorname{Re}=62,60 \%$. These values indicate that it is possible to predict, with $62.60 \%$ accuracy, that open complaints are filed without service. Accuracy, which reflects the quality of the algorithm, is $65.216 \%$, and the Recall, which reflects completeness, is at $62.596 \%$. The F-measure is $\mathrm{F}=0,64$, indicating that Precision and Recall are moderately accurate.

By analyzing the rules generated by the tree, it is possible to identify some interesting information, such as:

- $\quad$ Some complaints are never answered (Atendida = "N"), independently of the other attributes, such as "Line in Banks", "Innacurate Publicity" and "Non-Payment of Indemnity".

- Some complaints are always answered (Atendida $=$ "S"), independently of the other attributes, such as "Calculation of Installment Anticipation", "No Contract Copy Delivery" and "Arrears Calculation".

Figure 8 shows the trade-off between the True Positives ( $x$ axis) and True Negatives ( $y$ axis).

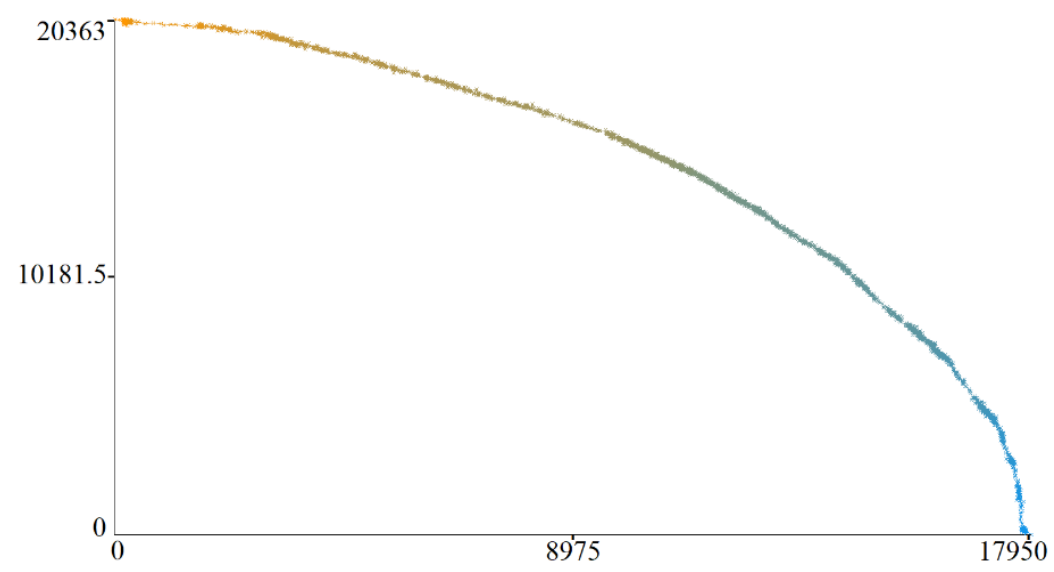

Figure 8. Relation between True Positives and True Negatives 
The generated decision tree is too large to be represented in full, but to illustrated the generated rules, 10 random rules were selected, as listed below (in Portuguese):

1. DescricaoProblema = Recusa Em Receber Pagamento De Nota Promissória, Carne, Etc.

- SexoConsumidor $=\mathrm{M}: \mathrm{N}(40.26 / 13.15)$

2. DescricaoProblema = Recusa Em Receber Pagamento De Nota Promissória, Carne, Etc.

- SexoConsumidor $=\mathrm{F}$

$-\mathrm{UF}=\mathrm{SP}: \mathrm{S}(5.02 / 1.01)$

3. DescricaoProblema = Cobrança Vexatória/Difamatória $-\mathrm{UF}=\mathrm{DF}$

- AnoCalendario ¿ 2013: S (4.0/1.0)

4. DescricaoProblema = Calculo De Encargos Na Cobrança De Cheque: S (31.2/12.09)

5. Descricaoproblema $=$ Sac - Resolução De Demandas (Ausência De Resposta, Excesso De Prazo, Não Suspensão Imediata Da Cobrança) $-\mathrm{UF}=\mathrm{SC}$

- SexoConsumidor $=\mathrm{M}$

- - - AnoCalendario ¿ 2013: S (23.1/5.02)

6. DescricaoProblema = Antecipação De Financiamento

$-\mathrm{UF}=\mathrm{SP}$

- FaixaEtariaConsumidor = entre 21 a 30 anos

- - AnoCalendario: 2013: S (5.43/2.14)

7. DescricaoProblema = Cobrança Indevida.

$-\mathrm{UF}=\mathrm{GO}$

- - FaixaEtariaConsumidor = mais de 70 anos

- - AnoCalendario ¿ 2014: S (14.11/5.19)

8. DescricaoProblema = Cobrança Indevida.

- UF = RJ: N (548.0/192.0)

9. Descricaoproblema = Cobrança Indevida.

$-\mathrm{UF}=\mathrm{SP}$

- AnoCalendario : 2014

- - FaixaEtariaConsumidor = entre 21 a 30 anos

- - - SexoConsumidor $=\mathrm{F}: \mathrm{S}$ (39.27/13.27)

10. DescricaoProblema = Consumidor Negativado Indevidamente Nos Serviços De Proteção Ao Credito

$-\mathrm{UF}=\mathrm{DF}$

- FaixaEtariaConsumidor = entre 41 a 50 anos

- - SexoConsumidor $=\mathrm{M}$

- - - AnoCalendario ¿ 2014: S (3.0/1.0)

As it is possible to observe, not all branches use all the attributes. The tree level was defined by the problem description, and the other attributes were used in an auxiliary way to generate rules.

\section{CONCLUSIONS AND FUTURE WORKS}

The SINDEC data used reflect the user satisfaction about products and services in Brazil from 2013 to 2017. The information extracted allows us to outline patterns of the most problematic market sectors, covered subjects and problems addressed. By studying the data using descriptive analysis, it is possible to understand how the data is distributed, and with the use of the decision tree classification technique, it is possible to predict what the result of a dataset is. In addition, by examining the rules created during training, we can find patterns that are normally invisible in another way.

As future work, other experiments with this database will be presented and complemented, using all attributes, and using other classification algorithms to identify new hidden patterns and evaluate quality indicators. 


\section{REFERENCES}

Ahishakiye, E., Taremwa, D., Omulo, E. O., and Niyonzima, I. (2017). Crime Prediction Using Decision Tree (J48) Classification Algorithm. International Journal of Computer and Information Technology, 06(03):2279-764.

Alippi, C. and Roveri, M. (2010). Virtual k-fold cross validation: An effective method for accuracy assessment. In The 2010 International Joint Conference on Neural Networks (IJCNN), pages 1-6. IEEE.

Bessa, L. R. and de Moura, W. J. F. (2014). Manual dos direitos do consumidor. Ministério da Justiça Secretaria Nacional do Consumidor Departamento de Proteção e Defesa do Consumidor, Brasilia, 4 edição edition.

Bevington, G. and Wolcott, H. F. (1996). Transforming Qualitative Data: Description, Analysis, and Interpretation. The Modern Language Journal, 80(3):405.

de Castro, L. N. and Ferrari, D. G. (2015). Introdução à mineração de dados: conceitos básicos, algoritmos e aplicações. Saraiva, São Paulo.

Ciaburro, G. and Venkateswaran, B. (2017). Neural Networks with R Smart models using CNN, RNN, deep learning, and artificial intelligence principles. Packt Publishing, Birmingham.

Claster, W. B., Dinh, H., and Cooper, M. (2010). Naive bayes and unsupervised artificial neural nets for caneun tourism social media data analysis. Proceedings - 2010 2nd World Congress on Nature and Biologically Inspired Computing, NaBIC 2010, pages 158-163.

Dwivedi, S. K. and Rawat, B. (2016). A review paper on data preprocessing: A critical phase in web usage mining process. Proceedings of the 2015 International Conference on Green Computing and Internet of Things, ICGCIoT 2015, pages 506-510.

Gerosa, L. (2017). Consumer Business Complaints in Brazil.

Jo, Y., Kim, E.-G., and Shin, Y. (2017). Graphical Keyword Service for Research Papers with Text-Mining Method. In Proceedings of the International Conference on Compute and Data Analysis - ICCDA '17, pages 185-190, New York, New York, USA. ACM Press.

Ministério da Justiça (2019). Cadastro Nacional de Reclamações Fundamentadas (PROCONS - Sindec).

Nazzal, J. M., El-emary, I. M., and Najim, S. a. (2008). Multilayer Perceptron Neural Network (MLPs) For Analyzing the Properties of Jordan Oil Shale. World Applied Sciences Journal, 5(5):546-552.

Nurhayati, Soekarno, I., Hadihardaja, I. K., and Cahyono, M. (2014). A study of hold-out and k-fold cross validation for accuracy of groundwater modeling in tidal lowland reclamation using extreme learning machine. In 2014 2nd International Conference on Technology, Informatics, Management, Engineering \& Environment, pages 228-233. IEEE.

Patil, T. and Sherekar, M. (2013). Performance Analysis of Naive Bayes and J48 Classification Algorithm for Data Classification. International Journal Of Computer Science And Applications, 6(2):256-261.

Quinlan, J. R. (1986). Induction of decision trees. Machine Learning, 1(1):81-106.

Qureshi, M. N., Aldheleai, H. F. H., and Tamandani, Y. K. (2015). An improved documents classification technique using association rules mining. In 2015 IEEE International Conference on Research in Computational Intelligence and Communication Networks (ICRCICN), pages 460-465. IEEE.

Rodriguez, J., Perez, A., and Lozano, J. (2010). Sensitivity Analysis of k-Fold Cross Validation in Prediction Error Estimation. IEEE Transactions on Pattern Analysis and Machine Intelligence, 32(3):569-575.

Samsani, S. (2016). An RST based efficient preprocessing technique for handling inconsistent data. In 2016 IEEE International Conference on Computational Intelligence and Computing Research (ICCIC), pages 1-8. IEEE.

Silva, L. A., Peres, S. M., and Boscarioli, C. (2016). Introdução à Mineração de Dados - Com Aplicação Em R. Campus - Grupo Elsevier, 1st edition.

Silva, L. M., Marques de Sá, J., and Alexandre, L. A. (2008). Data classification with multilayer perceptrons using a generalized error function. Neural Networks, 21(9):1302-1310. 